\author{
MRS Advances (C) 2018 Materials Research Society \\ DOI: 10.1557/adv.2018.331
}

\title{
Scaling Electrowetting with Printed Circuit Boards for Large Area Droplet Manipulation
}

\author{
Udayan Umapathi ${ }^{1}$, Samantha Chin ${ }^{2}$, Patrick Shin ${ }^{1,}$ Dimitris Koutentakis ${ }^{1}$, Hiroshi Ishii ${ }^{1}$ \\ ${ }^{I}$ MIT Media Lab, Cambridge, MA, U.S.A
}

${ }^{2}$ Wellesley College, MA, U.S.A

ABSTRACT

Droplet based microfluidics (digital microfluidics) with Electrowetting on dielectric (EWOD) has gained popularity with the promise of being technology for a true lab-on-chip device with applications spanning across assays/library prep, next-gen sequencing and point-of-care diagnostics. Most electrowetting device architecture are linear electrode arrays with a shared path for droplets, imposing serious limitations -- cross contamination and limited number of parallel operations. Our work is in addressing these issues through large $2 D$ grid arrays with direct addressability providing flexible programmability.

Scaling electrowetting to larger arrays still remains a challenge due to complex and expensive cleanroom fabrication of microfluidic devices. We take the approach of using inexpensive PCB manufacturing, investigate challenges and solutions for scaling electrowetting to large area droplet manipulation. $P C B$ manufactured electrowetting arrays impose many challenges due to the irregularities from process and materials used. These challenges generally relate to preparing the surface that interfaces with droplets -- a dielectric material on the electrodes and the top most hydrophobic coating that interfaces with the droplets. A requirement for robust droplet manipulation with EWOD is thin $(<10 u m)$ hydrophobic dielectric material which does not break down at droplet actuation voltages (AC/DC, $60 \mathrm{~V}$ to $200 \mathrm{~V})$ and has a no droplet pinning. For this, we engineered materials specifically for large area PCBs.

Traditionally, digital microfluidic devices sandwich droplets between two plates and have focussed on sub-microliter droplet volumes. In our approach, droplets are on an open surface with which we are able to manipulate droplets in microliter and milliliter volumes. With milliliter droplet manipulation ability on our electrowetting device, we demonstrate "digital millifluidics". Finally, we report the performance of our device and to motivate the need for large open arrays we show an example of running multiple parallel biological experiments. 


\section{INTRODUCTION}

Historically, automated liquid handling has relied on pressure differentials to transport liquid. In the 1990s, microfluidic liquid manipulation was developed following DARPA's push for field-deployable liquid handling systems. Microfluidics is the science and art of handling small volume fluids. Liquid handling devices in pharmaceuticals and gene synthesis require multiscale fluid handling ability. The state-of-the art systems are generally pressure driven systems connected with valves and tubes. These systems are manually assembled, expensive, and unreliable. With the growth of the genomic and drug industry we are moving towards more and more complex biological processes requiring smaller volume liquid manipulation capability. Manually assembled mechanical systems will not scale to the next generation of biological processes.

The electronics industry has demonstrated how to build robust integrated systems for information manipulation. With this as our motivation, we look towards electronics and integrated circuits to bring miniaturization, complexity and integration to enable the next generation of biology. The process of electrically moving liquid has existed since 1875 [1]. Since then, significant work has been done on using liquid in liquid electrowetting for paper like displays $[2,3]$. However, an even larger interest in the technique came from work using the technique for the automation of biology $[4,10]$. Application of EWOD for droplet microfluidics is popularly known as digital microfluidics $[4,10]$.

\section{Why scale electrowetting with printed circuit boards (PCB)?}

Most electrowetting devices in the literature use one dimensional row and column geometry for electrodes [5] or small grids [11]. This limitation arises primarily from the fabrication method used for making electrode arrays. The method of choice for fabricating electrodes involves clean rooms on substrates such as glass. Such fabrication methods have the advantage of smooth surface topography, no droplet pinning [14] and hence reliable motion of droplets at relatively lower actuation voltage $(<100 \mathrm{~V})$. However, glass and clean room processes are still expensive. Furthermore, the one dimensional geometry and small grids have some serious limitations. Multiple droplets share a common path, which leads to contamination between droplets. Additionally, operations must be run serially instead of in parallel. Expanding the geometry to a 2dimensional grid eliminates many of these problems with contamination and parallelization. A 2D grid architecture for an electrowetting device can be fabricated with multilayer routing methods such as in Integrated circuits and Printed Circuit Boards (PCBs). While integrated circuits offer the benefits of smooth surface topography needed in droplet manipulation device and miniaturization, for this work, we choose to focus on Printed Circuit boards owing to its low fabrication cost and quick turnaround time. It is important to note that some electrowetting devices with a 2D grid architecture still cannot parallelize because multiple electrodes share a common drive signal. The lack of individual addressability to each electrode limits the number of unique operations that can be executed in parallel and liquid handling potential of such devices.

\section{Open plate?}

For electrowetting droplet manipulation, a droplet can either be placed on an open surface (single plate) or sandwiched between two plates typically separated by 100 
$\mu \mathrm{m}-300 \mu \mathrm{m}$ (double plate). For this work, we chose to focus on single plate, since the open nature of the device enables easy addition/removal and efficient mixing of droplets.

\section{FABRICATING ELECTRODES WITH PRINTED CIRCUIT BOARDS}

We fabricated our electrowetting device using a standard PCB fabrication method. The electrodes were arranged to form a 2D grid as in Figure 1. For each electrode in our electrowetting device, a single electrical connection carries a high voltage to actuate

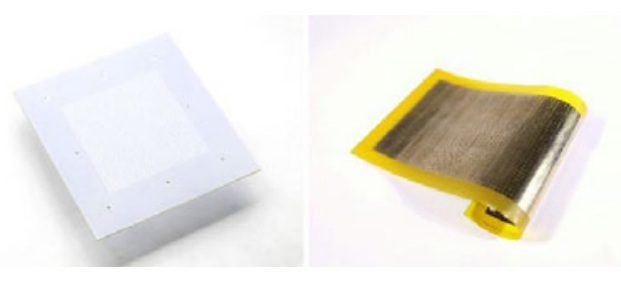

Figure 1. Left: Photograph of a 2D electrowetting square grid fabricated with standard PCB process on rigid FR4 substrate; measures 3.5" X 3.5" and consists of 1024 electrodes, each electrode measures $2.5 \mathrm{~mm}$ X $2.5 \mathrm{~mm}$. Right: Photograph of a 2D rectangular electrowetting grid (3.3" X 6.6") fabricated on flexible Pyralux PCB substrate; measures $3.5 "$ X 7" and consists of 2048 electrodes, each electrode measures $2.5 \mathrm{~mm} \mathrm{X} 2.5 \mathrm{~mm}$.

droplets and feedback signal for measuring the capacitance. These electrical connections are routed through 4 layers under the electrode grid.

PCB manufacturing supports a range of flexible and rigid substrates. We fabricated electrode grids on rigid (Figure 1, Left) and flexible (Figure 1, Right) substrates. Due to these advantages and the commodity nature of rigid PCB fabrication $(\$ 0.08 / \mathrm{cm} 2)$, we chose to fabricate all our electrowetting device on rigid PCBs. Using PCBs reduces cost of fabrication; PCBs are an attractive substrate for low-cost liquid handling. Electrowetting literature shows that manufacturing of non-pinning surfaces is the way to develop robust low voltage driven electrowetting devices. However, standard PCB fabrication process imposes several challenges in achieving this goal.

\section{OPTIMIZING SURFACE TOPOGRAPHY OF A PCB FOR REDUCED PINNING}

In a standard PCB process, the thickness of the outer layer of copper can range from $35 \mu \mathrm{m}$ to $70 \mu \mathrm{m}$. The separation between electrodes and connection vias introduce "canyons" which can pin droplets [14] and stop their motion. In a 1 oz. PCB process (1 ounce of copper rolled out to an area of 1 square foot), between electrodes the canyons are $140 \mu \mathrm{m}$ wide, $35 \mu \mathrm{m}$ deep as shown in Figure 2. Additionally, the vias in the electrodes introduce $250 \mu \mathrm{m} \mathrm{X} 35 \mu \mathrm{m}$ canyons. 

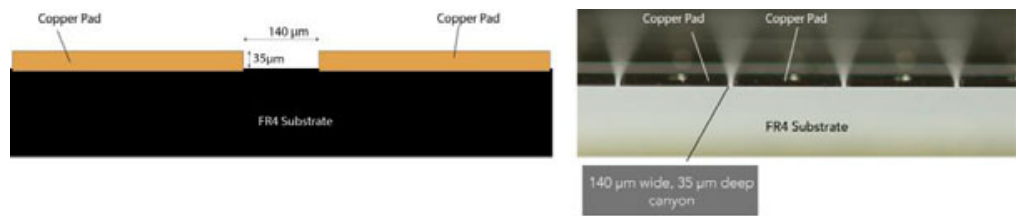

Figure 2. (Left) Canyon introduced by spacing between electrodes. Each canyon measures $140 \mu \mathrm{m} X 35 \mu \mathrm{m}$. Cross section of a rigid PCB showing rough topography of the surface of electrode grid. Canyons appear on the top surface from vias and inter-electrode spacing.

These canyons are surface imperfections that can reduce droplet mobility by pinning and increase the required droplet actuation voltage $[14,15]$. To mitigate effects of pinning from surface defects, we optimized the standard PCB fabrication process to reduce interelectrode spacing to $\sim 50 \mu \mathrm{m}$ and electrode height to $\sim 18 \mu \mathrm{m}$. The reduction in electrode spacing from $140 \mu \mathrm{m}$ to $50 \mu \mathrm{m}$ resulted in reduce of actuation voltage by $\sim 25 \mathrm{~V}$. With advanced PCB fabrication process it should be possible for further optimization.

\section{DIELECTRIC COATING:}

In EWOD, a layer of dielectric is applied to isolate the droplets from the electrodes - this prevents electrolysis. Most electrowetting devices in literature coat the PCB surface with Parylene-C and Silicon dioxide [6] in vapor deposition chambers. Typically these coatings measure only a few hundred nanometers and conform to the surface topography. This method works well for electrodes on glass since the surface imperfections from the electrodes are on the order of nanometers and do not affect the motion of large microliter droplets (no droplet pinning). Since the surface imperfections in PCBs are significantly larger (tens of micrometers), conformal coating methods which follow the topography of the PCB surface are not effective.

\section{Fabrication method to achieve smooth surface topography}

To achieve smooth surface topography, we first fill the canyons between electrodes with liquid photoimageable (LPI) soldermask during PCB manufacturing process (transparent). With the mask almost the canyon height $(\sim 18 \mu \mathrm{m})$, we achieve an approximately planar surface. We then heat bond membranes of porous Polytetrafluoroethylene (PTFE) or Parafilm M membrane. We bonded the $5 \mu \mathrm{m}$ thick PTFE membrane at $90^{\circ} \mathrm{C}$. In order to achieve uniform bonding without any wrinkles we pre-stretch the membrane. For the Parafilm, we stretch from its standard $130 \mu \mathrm{m}$ thickness up to its yield point $(\sim 40 \mu \mathrm{m})$ and then bond at $45^{\circ} \mathrm{C}$. The finished surface of an electrode grid does not reflect any roughness from the topography of the PCB even on large arrays (3.3" X 6.6”).

\section{Slippery coating for droplet motion}

Surface smoothness is not the only parameter required for successful droplet movement. Electrowetting surfaces must have high droplet mobility and adhere 
minimally to biological material. To achieve this, we infuse the membrane with low surface tension and low-viscosity ( $\sim 4 \mathrm{cSt})$ such as perflourinated oil or Silicone oil by spraying. Pressurized spraying makes the oil penetrate and retain it in the porous matrix of the membrane.

\section{DEVICE ARCHITECTURE}

The device of interest in this paper is built on the principles of Electrowetting on Dielectric (EWOD). A typical EWOD device consists of electrode array, a layer of dielectric, hydrophobic coating and electronics for actuation and sensing; see Figure 3. A microcontroller generates drive signals for the electrodes and process feedback obtained from the sensing unit as shown in Figure 3 (right). We multiplex the high voltage actuation $(65 \mathrm{~V}, 2 \mathrm{kHz})$ and a low voltage sensing signal $(3.3 \mathrm{~V}, 20 \mathrm{kHz})$. For high voltage, we use MOSFET arrays in push-pull configuration with individual drive signal on each electrode. We use touchscreen sensors for sensing location and correction of any failed droplet movement.
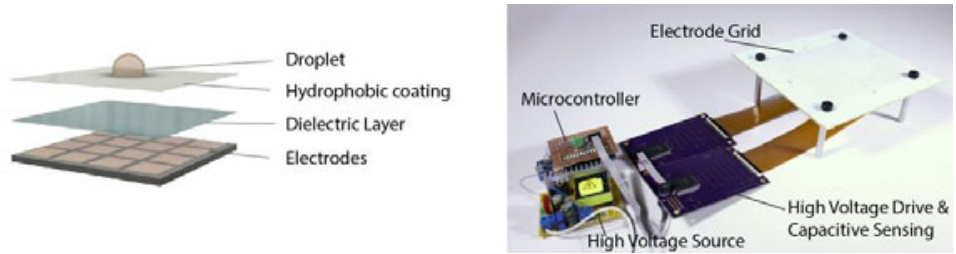

Figure 3. (Left) Layers of a typical electrowetting array. Droplet is positioned on an open surface. (Right) Overview of the electronics for high voltage actuation and capacitive sensing.

\section{DEVICE PERFORMANCE}

To characterize the performance of the device we conducted three sets of experiments: measuring droplet sliding angle, measuring contact angle change and droplet actuation (transport, merging and mixing).

\section{Measuring Droplet Sliding Angle}

Sliding angle is the surface tilt required for droplet motion and directly characterizes resistance to droplet mobility[13]. To evaluate this, we pipetted different volumes of DI water droplets on to the unactuated PCB and observed the angle at which they slid when the surface was tilted. This test was performed for PCB surface with liquid infused PTFE membrane, the results are comparable for other membranes. For large microliter droplets $(>10 \mu l)$ droplets become mobile at relatively low tilting angle ( $\sim 2.5$ degrees); see Figure 4 . Motion of droplets at low surface tilt is an indication that the surface largely does not inhibit droplet motion. 


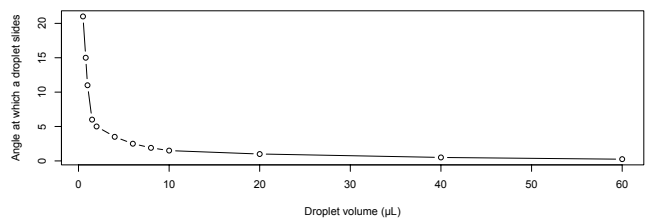

Figure 4. Sliding Angle as a function of droplet volume for liquid infused PTFE membrane.

\section{Measuring Contact Angle Change and Saturation}

For an immobile droplet, the wettability is measured in terms of the contact angle. Contact angle saturation [16] is major phenomena in electrowetting systems that can severely limit device performance [15]. For a droplet on an electrode, the contact angle is a function of the applied voltage as given by equation 1 ,

$$
\cos \theta_{v}=\cos \theta_{0}+1 / \gamma L G * 1 / 2 * 1 / \mathrm{d}^{*} \varepsilon * V^{2}
$$

$\theta_{0}$ is the contact angle when the electric field is zero (i.e. no voltage applied) and $\theta_{v}$ is the contact angle when a voltage $V$ is applied, $\gamma L G$ is the surface tension at the liquid-air interface and $\varepsilon$ is the dielectric constant of the film and $d$ is the thickness of the film between the electrode and the droplet. We inserted a platinum reference electrode similar to Moon et al [6] in a 50 $\mu 1$ DI water droplet with colored dye and varied the voltage up to $400 \mathrm{~V}$ (DC) to measure the contact angle change as shown in pictures of Figure 5 . We observed no contact angle saturation up to the input 400V for PTFE and Parafilm.

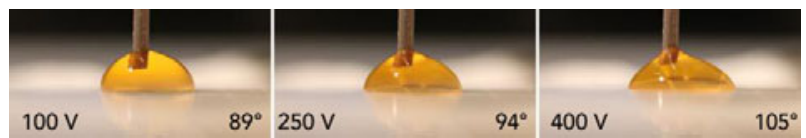

Figure 5. Contact angle of immobile water droplet as function of increasing voltage. We observed no contact angle saturation up to DC $400 \mathrm{~V}$ for PTFE.

\section{Droplet actuation: Transporting, Merging and Mixing}

By switching potential across electrodes, our electrowetting platform can actuate droplets - transporting a droplet (Figure 6, Left) and merging droplets (Figure 6, Right).

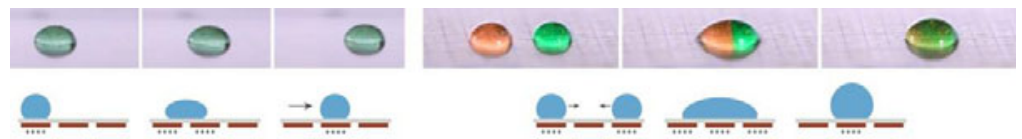

Figure 6. (Left) Droplet transport across the electrowetting surface. (Right) Sequence showing two droplets being merged. 
For PTFE membrane $(5 \mu \mathrm{m})$ a minimum actuation voltage of $50 \mathrm{~V}$ was observed for DI water droplets. We observed reliable repeated motion over all driving electrodes on our device at $90 \mathrm{~V}$. We achieved a maximum transport speed of $\sim 100 \mathrm{~mm} / \mathrm{s}$ with $50 \mu 1$ DI water droplet. Lowering the viscosity of the lubricating oil could improve droplet mobility.

We have successfully transported droplet volumes from $2 \mu 1$ to $1200 \mu 1$ ( $21 \mathrm{~mm}$ diameter). With synthetic biology as target application space, on our device we have transported droplets with DNA samples, DNA polymerases, buffers and dNTPs.

Droplets on an open surface mix efficiently in comparison to those between two plates due to larger diffusion rate and surface waves from collision[9]. For a preliminary analysis, we programmed our device to transport two colored droplets (each $50 \mu l$ ) on the same row of electrodes and collided them. From a camera footage, we observed the coalesced droplet until the two colors mix into a single uniform color to estimate the mixing time. As seen from results in Figure 7 (left), mixing time is low for droplets colliding at high speeds.
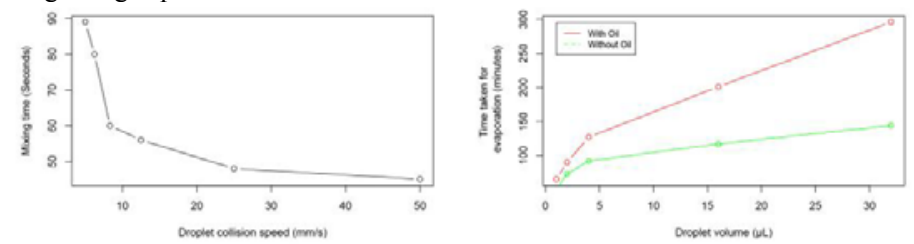

Figure 7. Left: Time taken for two colliding droplets to mix as a function of collision speeds. Right: Time taken for a droplet to evaporate entirely on a PTFE membrane with no oil (green) and with oil (red).

For electrowetting on an open surface, evaporation is a key concern. Figure 7 (right) shows the time taken for DI water droplets of various volumes to completely evaporate when placed on PTFE membrane without (Green) and with oil (Red). As seen from the plots, it takes longer for a droplet of given volume to evaporate on liquid infused surface. We noted that the reduced evaporation is due to oil surrounding the droplet at and just above the contact line (observed visually through a camera).

Figure 8 shows an illustrative experiment where four parallel stirring operations are being carried out to dilute DNA samples. On each corner a central stir operation is performed on a large droplet containing the original DNA sample. DI water droplets are then transported and merged sequentially in various quantities to create solutions of various concentrations. 

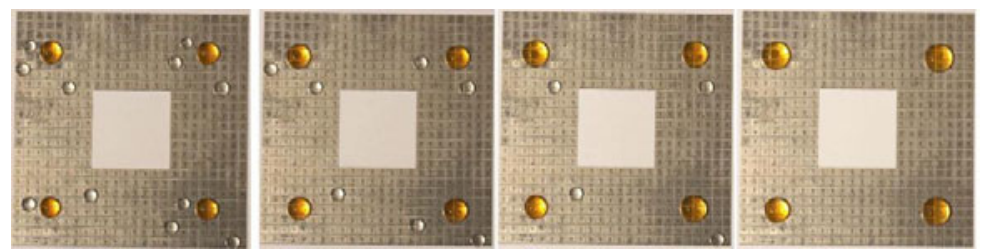

Figure 8. Photographs of 4 parallel operations being carried out in parallel. Droplets of $10 \mu$ to $20 \mu l$ are being transported and merged in to the central $50 \mu l$ droplet. The 4 large droplets (colored droplets) being stirred contain samples of DNA molecules. The end result of the mixing and stirring is creation of solutions of various dilutions.

\section{FUTURE - MULTISCALE DIGITAL FLUIDICS AND INTEGRATION:}

On our electrowetting platform we were able to move, merge and mix droplet volumes from $2 \mu \mathrm{l}$ to $1200 \mu \mathrm{l}$. We have demonstrated digitally manipulating droplets of volumes two orders of magnitude apart. This shows the possibility of performing multiscale liquid manipulation digitally on a single electrowetting device. Liquid handling devices in pharmaceuticals and gene synthesis require multiscale fluid handling ability. The state-of-the art systems are almost entirely pressure driven; these systems use valves and tubes of different sizes to transition from millifluidics to microfluidics and hence cumbersome to assemble and expensive. We believe electrowetting systems have the potential to economically scale to compete with existing pressure driven systems.

In addition to droplet manipulation, electronic systems can perform a range of actuation (Ex: temperature control) and sensing (Ex: optical detection). Droplet manipulation (liquid handling) is part of the problem in scaling up lab-on-chip technologies. Economic scaling of integrated functionalities for other actuation and sensing mechanism is desirable and we leave this as future work.

\section{CONCLUSION}

While digital microfluidics based on electrowetting has the potential to be the technology for true lab-on-chip devices, scaling it economically has remained a challenge. We believe that electrowetting droplet manipulation can scale to manipulate large number of droplets on a single chip if scaled economically. With this goal, our work looked at fabricating electrowetting lab-on-a-chip devices on inexpensive printed circuit boards and described how to overcome the challenges imposed by PCB manufacturing process. We also demonstrated how on a single device droplet volumes of two different orders of magnitude can be manipulated on the same device. This hints at the possibility of multiscale integrated digital fluidics for biology.

\section{ACKNOWLEDGMENT}

The authors would like to thank Neil Gershenfeld, Joseph M. Jacobson, Rui Qing, Prashant Patil, Sam Calisch and other members of Center for Bits and Atoms 
(MIT). We also thank Amos Golan, Carolynn Will and other members of Tangible Media (MIT Media Lab), Ryan Fobel and Urs Gaudenz for their support in various stages of this work.

\section{REFERENCES}

1. Gabriel Lippmann, PhD. Thesis, Gauthier Villars, 1875.

2. Liquavista. Available at: https://www.liquavista.com/ (accessed 20 November 2017).

3. G. Beni and S. Hackwood, Applied Physics Letters 38(4), 207-209 (1981).

4. M.G. Pollack, R.B. Fair, and A.D. Shenderov, Applied Physics Letters 77(11), 1725-1726 (2000).

5. N. Mei, B. Seale, A.H. Ng, A.R. Wheeler, and R. Oleschuk, Analytical Chemistry, 86(16), 84668472 (2014).

6. H. Moon, S.K. Cho, R.L. Garrell, and C.J.C. Kim, Journal of applied physics, 92(7), 4080-4087 (2002).

7. P.W. Wilson, W. Lu, H. Xu, P. Kim, M. J. Kreder, J. Alvarenga, and J. Aizenberg, Physical Chemistry Chemical Physics, 15(2), 581-585 (2013).

8. E. Seyrat and R.A. Hayes, Journal of Applied Physics, 90(3), 1383-1386 (2001).

9. C.G. Cooney, C.Y. Chen, M.R. Emerling, A. Nadim. and J.D. Sterling. Microfluidics and Nanofluidics, 2(5), 435-446 (2006).

10. M.L. Jebrail, A.R. Wheeler. Current opinion in chemical biology, 14(5), 574-81 (2010).

11. M. Alistar, U. Gaudenz. Bioengineering, 4(2), 45(2017).

12. D.G. Rackus, R.P. de Campos, C. Chan, M.M. Karcz, B. Seale, T. Narahari, C. Dixon, M.D. Chamberlain, A.R. Wheeler. Lab on a Chip (2017).

13. W. Chen, A.Y. Fadeev, M.C. Hsieh, D. Öner, J. Youngblood, and T.J. McCarthy. Langmuir, 15(10), 3395-3399 (1999).

14. P.G. De Gennes. Reviews of modern physics, 57(3), 827 (1985).

15. S.W. Walker, B. Shapiro, R.H. Nochetto. Physics of Fluids. 21(10), 102103 (2009).

16. H. J. I. Verheijen and M. W. J. Prins. Langmuir, 15(20), 6616-6620 (1999). 